\section{Inequidad en la utilización de servicios de salud reproductiva en Colombia en mujeres indígenas y afrodescendientes}

\author{
Ethnic disparities in the use of reproductive health \\ services by indigenous and African-descendant \\ Colombian women
}

\author{
Disparidades étnicas no uso de serviços de saúde \\ reprodutiva por mulheres colombianas \\ indígenas e afrodescendentes
}

\begin{abstract}
The aim of this study in Colombia was to analyze the use of reproductive health services (prenatal care, childbirth, and postpartum) according to women's ethnicity, based on the National Demographic and Health Survey for 2010. Ethnicity was self-reported (indigenous, African-descendant, or neither), and logistic models were performed for each service. The use of reproductive health services was lower among indigenous and African-descendant Colombian women when compared to those that did not declare their ethnicity. According to the regression models, indigenous women showed lower odds of having an adequate number of prenatal visits $(O R=0.61)$, and the same was true for African-descendant women. The same situation prevailed for institutional childbirth care: indigenous women $(O R=0.33)$ and African-descendant women $(O R=0.60)$; and postpartum follow-up: indigenous $(\mathrm{OR}=0.80)$, African-descendant $(\mathrm{OR}=0.80)$. In conclusion, there are inequities in the use of reproductive health services by women belonging to ethnic groups in Colombia, who should be targeted with government strategies to guarantee their right to health.
\end{abstract}

Health Inequalities; Reproductive Health Services; Ethnic Groups; Reproductive Rights
Camilo Noreña-Herrera 1,2

René Leyva-Flores 2

Lina Sofía Palacio-Mejía 2

María Beatriz Duarte-Gómez ${ }^{2}$

\section{Resumen}

Se analizó la utilización de servicios de salud reproductiva (atención prenatal, parto y posparto), según la etnia de las mujeres, a partir de la Encuesta Nacional de Demografía y Salud 2010 de Colombia. La etnia fue medida por autoreconocimiento (indigenas, afrodescendientes y ninguna) y se estimaron modelos logísticos para cada servicio. Encontramos que, respecto a quienes no pertenecen a ninguna etnia, la utilización de servicios de salud reproductiva es menor en las mujeres indígenas y afrodescendientes. En los modelos de regresión se encontró que mujeres indigenas tienen menores posibilidades de un número adecuado de controles prenatales $(O R=0,61)$, así como también afrodescendientes. Igual situación para la atención institucional del parto: indígenas $(O R=0,33)$, afrodescendientes $(O R=0,60)$; y en control posparto: indígenas $(O R=0,80)$, afrodescendientes $(O R=$ 0,80). En conclusión, existen inequidades en la utilización de servicios de salud reproductiva en las mujeres de grupos étnicos de Colombia que deben ser atendidas con estrategias gubernamentales que garanticen el derecho a la salud.

Desigualdad en la Salud; Servicios de Salud Reproductiva; Grupos Étnicos;

Derechos Reproductivos 


\section{Introducción}

La salud sexual y la salud reproductiva, específicamente la salud materna, es considerada una prioridad global, siendo la reducción de la mortalidad materna en $75 \%$ y el acceso universal a la salud reproductiva las metas planteadas en el quinto Objetivo de Desarrollo del Milenio para el año 2015 1. Sin embargo, la tasa de mortalidad materna solo se redujo $45 \%$, a nivel mundial ${ }^{2}$. En la región de las Américas persisten importantes inequidades. La Organización Panamericana de la Salud (OPS) estimó para América Latina y el Caribe 6.040 defunciones maternas, expresada en una Razón de Mortalidad Materna (RMM) de 85 defunciones por cada 100.000 nacidos vivos, cifra mayor que en América del Norte (RMM = $27 / 100.000$ nacidos vivos) 3 . En el caso de Colombia, según la Organización Mundial de la Salud (OMS) para el 2013 el país tenía una de las tasas de mortalidad materna más alta de Sur América (83 muertes/100.000 nacidos vivos), distante de la meta para el 2015 (45 muertes/100.000 nacidos vivos) 4 .

El acceso y la utilización de servicios de salud reproductiva durante el proceso de gestación, parto y posparto son factores que contribuyen a la reducción de la mortalidad materna y perinatal. Durante los controles prenatales, mínimo cuatro recomendados por la OMS 5, es posible la identificación temprana de riesgos y complicaciones que deben ser tenidas en cuenta al momento del parto, el cual debe ser atendido por personal cualificado y al interior de los servicios de salud, considerando la posibilidad de complicaciones obstétricas que pueden presentarse en $15-20 \%$ de las embarazadas, aun en aquellas calificadas como de bajo riesgo durante el control prenatal ${ }^{6}$. Además, la atención inmediata del posparto y un adecuado seguimiento contribuyen a la reducción de mortalidad por infecciones y hemorragias, promueve la vacunación del recién nacido, la lactancia materna y permite identificar tempranamente la depresión posparto 7 .

La etnia ha sido reconocida como un determinante social de las inequidades en salud 8 , debido a las desigualdades injustas y evitables que se presentan en materia de salud y derechos sociales en las personas pertenecientes a grupos étnicos en comparación con la población general. Estas inequidades se originan en las estructuras políticas y socioeconómicas de las sociedades, y determinan las condiciones de vida y de trabajo de las personas ${ }^{9}$. Se ha documentado que el lugar de residencia de las comunidades étnicas se ubica en zonas rurales o periféricas de las ciudades, de difícil acceso, deficientes servicios públicos (agua potable, alcantarillado, electricidad), y ofer- ta de servicios de salud limitada. Solo el $30 \%$ de las personas cuenta con empleo remunerado 10 .

Para los grupos étnicos, sus diferencias culturales se convierten en barreras y motivo de discriminación en los servicios de salud y en la decisión de utilizarlos. En Guatemala, el no hablar el idioma español, se asoció con menor atención institucional del parto y de uso de métodos anticonceptivos modernos 11. En México y Guatemala se ha identificado que el riesgo de muerte materna es tres veces más alto para las mujeres indígenas ${ }^{12}$. En Bolivia se ha encontrado que existe una preferencia por la medicina y los métodos anticonceptivos tradicionales 13. Las mujeres indígenas de países con alto porcentaje de población indígenas como Paraguay, Bolivia y Guatemala tienen una tasa global de fecundidad superior a la media latinoamericana de 2,7 hijos 12,14 .

En Colombia la situación es similar. En 2010 en los municipios con alta población indígena de la Orinoquia, la Amazonia y el Pacífico, el porcentaje de mujeres embarazadas que tuvo cuatro o más controles prenatales sólo llegó a 30\%, mientras que el promedio nacional fue de $88,6 \% 15$. En 2011, 21\% de las mujeres indígenas de la ribera del Amazonas no recibió control prenatal 16. En la mayoría de los pueblos indígenas y las comunidades afrodescendientes de la costa pacífica, han sido reportadas barreras geográficas (distancias, escasez de transporte), económicas y culturales para la utilización de los servicios de salud 17 .

\section{El contexto colombiano}

Colombia es un Estado social de derecho, organizado en forma de República unitaria, y la administración está en gran parte descentralizada entre los 32 departamentos y 1.121 municipios que lo conforman. De acuerdo con el último censo de población del 2005, la población es de 41 millones, existiendo cinco grupos étnicos que representan el $14 \%$ de la población: afrocolombianos $(10,3 \%)$, pueblos indígenas $(3,4 \%)$, raizales $(0,1 \%)$, palenqueros $(0,02 \%)$ y rom o gitanos $(0,01 \%) 18$.

La comunidad Palenquera es afrodescendiente, pero tiene su propio idioma (palenquero), y residen en su mayoría en el departamento de Bolívar. Los raizales del Archipiélago de San Andrés, isla caribeña colombiana, son de raíces afrodescendientes, su idioma es el bandé y el inglés y su religión originaria la protestante. Ambos, grupos son considerados para el censo de población como población afrodescendiente. Además de estos dos grupos afrodescendientes, el país reconoce la existencia de cincuenta muni- 
cipios con territorios colectivos de comunidades negras; la región Atlántica y Pacífica son las de mayor porcentaje de población afrocolombiana 19. Existen 87 pueblos indígenas, la mayoría ubicados en el área rural de la Orinoquía y Amazonía, la región Pacífica, y la Atlántica y otros dentro de los veintisiete resguardos indígenas legalmente constituidos 18 .

Colombia posee un sistema de salud basado en un modelo de seguridad social bajo principios de equidad y universalidad que pretende a través del aseguramiento garantizar el acceso a los servicios de salud. Para cumplir con este propósito el sistema tiene dos regímenes de aseguramiento; el contributivo, que cubre a quienes tienen capacidad de pago o trabajo formal; y el subsidiado, para personas con bajos ingresos o trabajo informal, pagado por el gobierno, dentro del cual se encuentran las comunidades indígenas (Ley 100 de 1993 20). Los servicios de salud durante el embarazo y el parto son gratuitos para el régimen subsidiado 21 (Resolución 3.384 del 2000). Si bien existe una legislación especial en salud para estos grupos étnicos, que incluye la consulta sobre los programas y la creación de empresa indígenas para el aseguramiento y la prestación de servicios de salud, el acceso real a los servicios es deficiente y fraccionado y no cumple con los requisitos de calidad, interculturalidad, integralidad, aceptabilidad y acceso 22 . Concurren además problemas de salud relacionados con determinantes estructurales, como la desnutrición crónica en la población infantil 15,23.

Aunque el acceso a los servicios de salud reproductiva contribuye a garantizar los derechos reproductivos 24, en Colombia existen inequidades en la utilización de estos servicios en los grupos con menor nivel socioeconómico y educativo, desplazados por la violencia y pertenecientes a grupos étnicos relacionadas por obstáculos económicos, geográficos sociales y culturales 17,25,26. Un análisis de la Encuesta Nacional de Demografía y Salud (ENDS 2010) reportó como obstáculos para el control prenatal estos factores, más la falta de confianza en los prestadores, con diferencias regionales, aunque no estratifica sus resultados de acuerdo a la variable etnia 27 . La ENDS 2005 ya había reportado mayores porcentajes de mujeres sin control prenatal, sin atención del parto en los servicios de salud, y sin atención posparto en aquellas sin educación, residentes en el área rural, y con menor nivel socioeconómico 28 .

\section{La etnia como categoría de análisis}

La condición étnica contiene una dimensión histórica, permite reconocer a la sociedad en grupos con identidades propias y diferenciadas, que implica la construcción de una conciencia colectiva en torno a elementos como el idioma, la ascendencia y el territorio de origen, aunque el grupo ya no los ocupe 10,29. Actualmente, la caracterización étnica de las poblaciones constituye un aspecto de interés en el campo de las ciencias de la salud ${ }^{30}$, y ha sido fuente de debate, debido a la utilización y el peso dado en los análisis de estudios epidemiológicos y la forma de medición o identificación de dicha condición 29,31,32.

Una revisión sistemática sobre el uso de la variable etnia encontró que el principal método de identificación de la etnia fue la autoidentificación 31. El cual es cuestionado debido al subreporte dado en indígenas y afrodescendientes por motivos de discriminación 32 . En Colombia la identificación de la etnia se hace a través del autoreporte en una de las preguntas del censo de población, la cual clasifica a las personas en seis categorías: indígena, rom, raizal del archipiélago de San Andrés y Providencia, palenquero de San Basilio, afrodescendiente y ninguna 18 .

En este estudio entendemos la etnia como una condición social, teniendo en cuenta sus componentes histórico, cultural y socioeconómico. La realización de este análisis cuyo núcleo central es la etnia obedece a la necesidad de evidenciar posibles inequidades en la utilización de los servicios de salud reproductiva de las mujeres de los grupos étnicos del país. Considerando que en Colombia, al igual que varios países de América Latina, únicamente hasta los censos de población del año 2005, fue incluida la pregunta de auto-identificación étnica 33. La ENDS sólo incluyó la variable en su última versión del 2010, pero no fue usada para el análisis ni en el reporte de los resultados generales de la Encuesta 34 .

Considerando este panorama, el presente estudio tiene como objetivo examinar la asociación entre la etnia y la utilización de los servicios de salud institucionales de control prenatal, atención del parto, y el control posparto, a través del análisis de la ENDS 2010.

\section{Material y métodos}

\section{Muestra}

Se realizó un análisis de la información de salud reproductiva generada por la ENDS 2010, realizada cada cinco años desde 1990 por Profamilia, organización privada sin ánimo de lucro. La en- 
cuesta tiene cobertura nacional, es probabilística, representativa de las zonas urbanas y rurales, y divide el país en departamentos (primeras divisiones administrativas), regiones y subregiones 34 . La ENDS 2010 realizó un muestreo estratificado multi-etápico tipo clúster, que incluyó 51.447 hogares, ubicados en las zonas urbanas y rurales de 258 municipios, con una tasa de respuesta de $92 \%$. El número de mujeres en edad fértil (13-49 años) efectivamente entrevistadas llegó a 53.521 y la tasa de respuesta fue de $94 \%$. Dicha encuesta indaga acerca de las características de la atención prenatal, parto y posparto, relacionadas con el último hijo(a) nacido(a).

La muestra de elegibles para el presente análisis fue de 14.521 mujeres con un hijo(a) nacido(a) a partir de noviembre de 2004. Esta cantidad incluía indígenas; gitanas; raizales; palenqueras; afrodescendientes; y las que no se autoreconocieron en ninguna etnia. Las palenqueras (tres), y raizales (ciento veinte), fueron incluidas en el grupo de afrodescendientes. Las gitanas (siete), no fueron consideradas en este análisis debido a su reducido tamaño de muestra. Se concluyó con una muestra de análisis de 14.514 mujeres (indígenas 1.962; afrodescendientes 1.729; sin pertenencia étnica 10.823).

\section{Variables dependientes}

\section{- Control prenatal del último nacido}

Se preguntó: “¿Cuándo usted estaba embarazada se hizo control prenatal alguna vez?”, con las opciones sí/no como respuesta. Con el fin de categorizar y calificar el cumplimiento de la norma colombiana respecto al número mínimo de controles prenatales se construyó el índice de utilización de control prenatal propuesto por Kotelchuck ${ }^{35}$, el cual está compuesto de un algoritmo que considera dos dimensiones: el bimestre de gestación de la primera atención prenatal y el porcentaje de atenciones recibidas (observadas), con respecto a las diez normadas (esperadas) por la guía de práctica clínica para la atención prenatal en Colombia 21. La razón entre las visitas observadas y esperadas se agrupó en cuatro categorías (menor del 50\%, entre 50\% y 79\%, entre 80 y $109 \%$ e igual o superior al $110 \%$ ). Finalmente, las dos dimensiones son combinadas mediante una sumatoria para la construcción de un índice de atención prenatal con cuatro categorías: inadecuado, intermedio, adecuado, adecuado plus. Las mujeres sin control prenatal o tardío (después del $4^{\circ}$ mes) fueron agrupadas en la categoría de inadecuado. Las categorías adecuado y adecuado plus ( $80 \%$ o más de las consultas normadas y antes del $2^{\circ}$ trimestre) fueron integradas en una sola, debido a porcentajes inferiores al $1 \%$ en cada categoría (Figura 1).

\section{- Atención institucional del parto}

Se preguntó: ¿Dónde tuvo lugar el parto? y se recodificaron las opciones de respuesta en dos categorías: atención institucional $=1$, en caso de que el parto haya sido en hospital, clínica institución o consultorio médico particular. No atención institucional $=0$, cuando ocurrió en el propio hogar u otro hogar, ya fuera por partera empírica o sin ayuda.

\section{- Control posparto}

La pregunta fue: “ $¿$ Tuvo usted algún control médico después del parto?” (sí = 1/no=0).

\section{Variables independientes}

La variable independiente de mayor interés fue la etnia, reportada a través de la auto identificación con la pregunta: “ $¿$ De acuerdo con su cultura, pueblo o rasgos físicos, usted es o se reconoce como: indígena, gitana, raizal, palenquera, afrodescendiente, o ninguna de las anteriores?" Para el análisis se tuvo en cuenta tres grupos: indígenas, afrodescendientes y aquellas que no se reconocen en ninguna etnia.

Como se observa en la Tabla 1, se analizaron variables sociodemográficas de la mujer y el hogar como: edad; nivel de escolaridad; estado civil; tipo de familia: unipersonal (mujer sola); nuclear (conformada por mujer y hombre, con o sin hijos), extensa (hogar conformado por una familia nuclear más otros parientes no-nucleares, exclusivamente) y compuesta (familia formada por una familia nuclear o una familia extensa más otros no-parientes); jefatura del hogar; ocupación; índice económico del hogar dado en quintiles, construido a través de una serie de bienes de consumo durables, características de la vivienda y de acceso a servicios básicos 36 , región y zona de residencia. También se tomaron en cuenta antecedentes de la madre como hospitalización durante el embarazo y antecedente de mortalidad infantil.

\section{Análisis estadístico}

Exploramos la distribución de las variables en la totalidad de la muestra, y por grupos de comparación, con tablas de frecuencia, incluyendo intervalos del 95\% de confianza (IC95\%), y medidas de tendencia central y de dispersión. Mediante un modelo de regresión logística ordinal (modelo proporcional de odds ratio - OR) con variable de- 


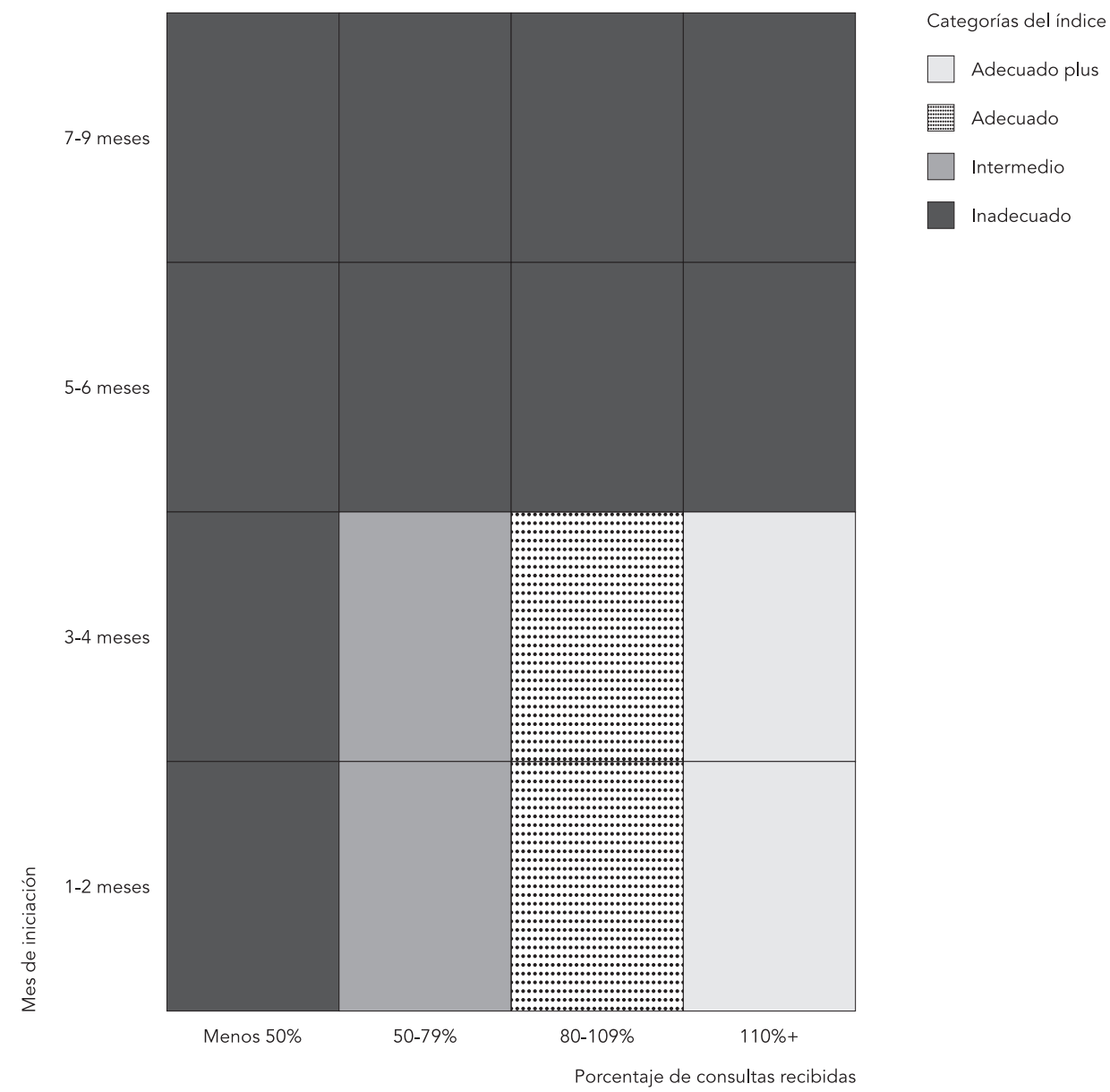

* Ajustado según la guía colombiana. Figura elaborada tomando como referencia Kotelchuck 34.

pendiente, el índice de atención prenatal en escala Likert (inadecuado, intermedio, adecuado/ plus) comprobamos su asociación con la etnia (indígenas, afrodescendientes versus no etnia), ajustando con las variables antes descritas. El modelo fue evaluado con el test de Brant para regresiones paralelas. Por otro lado, se ajustaron dos modelos de regresión logística binomial, uno para la atención del parto en los servicios de salud, y otro para la atención posparto, para comprobar su asociación con la etnia. Para estos modelos se evaluó la prueba de bondad de ajuste ${ }^{37}$, y el factor de inflación de la varianza. Todos los análisis fueron realizados con Stata 13 (StataCorp LP, College Station, Estados Unidos), usando el comando svy para encuestas complejas.
La hipótesis de estudio fue que ser indígena o afrodescendiente está asociado de manera inversa con la utilización de servicios de salud para la atención prenatal, del parto y posparto.

\section{Resultados \\ Características sociodemográficas}

De las 14.514 mujeres incluidas en el estudio, $13,5 \%$ se autoreconocieron como indígenas y $11,9 \%$ como afrodescendientes; el porcentaje restante no se reconocieron en ningún grupo étnico colombiano (74,6\%). La Tabla 1 muestra inequidades en los grupos étnicos en el ámbi- 
Tabla 1

Características sociodemográficas de mujeres de 13 a 49 años de acuerdo a su etnia *.

\begin{tabular}{|c|c|c|c|c|c|c|c|c|}
\hline & \multicolumn{2}{|c|}{$\begin{array}{c}\text { Total } \\
(\mathrm{N}=14.514)\end{array}$} & \multicolumn{2}{|c|}{$\begin{array}{l}\text { Indigena } \\
(n=1.962)\end{array}$} & \multicolumn{2}{|c|}{$\begin{array}{l}\text { Afrodescendiente } \\
\qquad(n=1.729)\end{array}$} & \multicolumn{2}{|c|}{$\begin{array}{c}\text { Ninguna } \\
(\mathrm{n}=10.823)\end{array}$} \\
\hline & $\%$ & IC95\% & $\%$ & IC95\% & $\%$ & IC95\% & $\%$ & IC95\% \\
\hline Edad media (años) [ES] ** & 28 & 0,1 & 28 & 0,3 & 28 & 0,2 & 28 & 0,1 \\
\hline Años promedio de educación [ES] ** & 9 & 0,1 & 6 & 0,2 & 9 & 0,15 & 9 & 0,1 \\
\hline \multicolumn{9}{|l|}{ Nivel de escolaridad ** } \\
\hline Sin educación & 1,8 & $1,5-2,1$ & 10,4 & $7,5-14,5$ & 3,0 & $2,1-4,1$ & 1,1 & $0,9-1,4$ \\
\hline Primaria & 23,7 & $22,6-24,8$ & 41,6 & $37,5-45,7$ & 25,0 & $22,2-28,0$ & 22,5 & $21,4-23,7$ \\
\hline Secundaria & 54,6 & $53,4-55,8$ & 38,3 & $33,9-42,9$ & 54,0 & $50,8-57,1$ & 55,6 & $54,2-56,9$ \\
\hline Superior & 20,0 & $18,9-21,0$ & 9,7 & $7,23-12,8$ & 18,0 & $15,6-20,7$ & 20,8 & $19,7-22,0$ \\
\hline \multicolumn{9}{|l|}{ Estado civil ** } \\
\hline Soltera & 26,6 & $25,6-27,6$ & 21,6 & $18,5-25,0$ & 28,0 & $25,3-30,8$ & 26,7 & $25,6-27,8$ \\
\hline Casada & 19,3 & $18,3-20,2$ & 14,6 & $11,8-17,9$ & 13,3 & $11,3-15,6$ & 20,4 & $19,3-21,5$ \\
\hline Unión libre & 53,0 & $51,9-54,2$ & 62,8 & $58,7-66,8$ & 57,7 & $54,8-60,6$ & 51,8 & $50,6-53,1$ \\
\hline Viuda & 0,9 & $0,7-1,1$ & 1,0 & $0,5-2,2$ & 0,8 & $0,5-1,5$ & 0,9 & $0,7-1,5$ \\
\hline Divorciada & 0,2 & $0,1-0,4$ & 0,0 & 0 & 0,2 & $0,0-1,0$ & 0,3 & $0,2-0,4$ \\
\hline \multicolumn{9}{|l|}{ Tipo de familia ** } \\
\hline Unipersonal & 0,2 & $0,1-0,3$ & 0,1 & $0,0-0,5$ & 0,2 & $0,1-0,5$ & 0,2 & $0,1-0,3$ \\
\hline Nuclear & 52,9 & $51,7-54,1$ & 53,0 & $48,8-57,2$ & 48,3 & $45,2-51,3$ & 53,5 & $52,2-54,8$ \\
\hline Extensa & 42,1 & $41,0-43,3$ & 40,8 & $36,8-45,0$ & 46,0 & $43,0-49,1$ & 41,7 & $40,4-43,0$ \\
\hline Compuesta & 4,8 & $4,3-5,3$ & 6,1 & $4,2-8,7$ & 5,5 & $4,2-7,1$ & 4,6 & $4,1-5,2$ \\
\hline \multicolumn{9}{|l|}{ 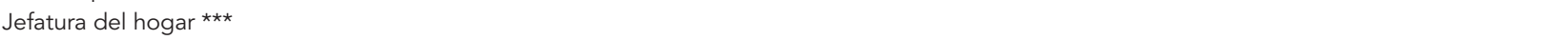 } \\
\hline Hombre & 70,8 & $69,7-71,9$ & 76,0 & $72,1-79,6$ & 69,1 & $66,0-72,1$ & 70,7 & $69,6-71,9$ \\
\hline Mujer & 29,2 & $28,2-30,7$ & 24,0 & $20,4-27,9$ & 30,9 & $27,9-34,0$ & 29,3 & $28,1-30,4$ \\
\hline \multicolumn{9}{|l|}{ Ocupación laboral ** } \\
\hline No trabaja & 13,2 & $12,4-14,0$ & 19,9 & $16,5-23,7$ & 15,9 & $13,9-18,2$ & 12,4 & $11,6-13,3$ \\
\hline Profesional/Técnico & 6,7 & $6,1-7,3$ & 3,8 & $2,4-5,9$ & 5,1 & $4,0-6,5$ & 7,1 & $6,4-7,8$ \\
\hline Oficinista & 7,6 & $7,0-8,3$ & 6,0 & $4,2-8,6$ & 5,3 & $4,1-6,9$ & 8,0 & $7,3-8,8$ \\
\hline Ventas y servicios & 62,6 & $61,4-63,8$ & 46,5 & $42,1-51,0$ & 62,5 & $59,5-65,4$ & 63,5 & $62,2-64,8$ \\
\hline Agricultura & 4,8 & $4,3-5,3$ & 16,1 & $12,3-20,7$ & 6,2 & $4,7-8,2$ & 3,9 & $3,5-4,5$ \\
\hline Manual & 5,2 & $4,7-5,8$ & 7,7 & $5,6-10,5$ & 4,9 & $3,7-6,5$ & 5,1 & $4,5-5,7$ \\
\hline \multicolumn{9}{|l|}{ Filiación en salud ** } \\
\hline No afiliada & 11,2 & $10,5-11,9$ & 9,6 & $7,6-12,0$ & 13,3 & $11,5-15,3$ & 11,0 & $10,3-11,8$ \\
\hline Subsidiado & 50,6 & $49,3-51,9$ & 76,4 & $72,5-79,9$ & 58,3 & $55,2-61,3$ & 48,1 & $46,6-49,5$ \\
\hline Contributivo & 36,1 & $34,8-37,3$ & 12,9 & $10,1-16,4$ & 26,2 & $23,4-29,2$ & 38,7 & $37,4-40,1$ \\
\hline Especial & 2,1 & $1,9-2,5$ & 1,1 & $0,5-2,2$ & 2,2 & $1,5-3,2$ & 2,2 & $1,9-2,6$ \\
\hline \multicolumn{9}{|l|}{ Índice de riqueza hogar ** } \\
\hline Más bajo & 22,8 & $21,6-24,1$ & 61,2 & $55,7-66,3$ & 37,0 & $33,2-40,9$ & 18,7 & $17,41-20$ \\
\hline Bajo & 22,6 & $21,4-23,8$ & 16,9 & $13,8-20,5$ & 23,8 & $21,1-26,9$ & 22,7 & $21,5-24,0$ \\
\hline Medio & 22,8 & $21,7-24,0$ & 12,7 & $10,0-16,0$ & 18,2 & $15,6-21,2$ & 24,0 & $22,8-25,4$ \\
\hline Alto & 18,6 & $17,6-19,7$ & 6,1 & $4,0-9,2$ & 13,6 & $11,3-16,2$ & 20,1 & $18,9-21,3$ \\
\hline Más alto & 13,2 & $12,2-14,2$ & 3,2 & $1,8-5,5$ & 7,4 & $5,8-9,4$ & 14,5 & $13,4-15,7$ \\
\hline \multicolumn{9}{|l|}{ Región de ubicación ** } \\
\hline Atlántica & 22,0 & $21,1-23,0$ & 35,1 & $29,7-41,0$ & 32,5 & $29,0-36,1$ & 19,8 & $18,8-20,8$ \\
\hline Oriental & 18,9 & $18,0-19,8$ & 3,0 & $1,89-4,72$ & 6,2 & $4,70-8,14$ & 21,6 & $20,6-22,6$ \\
\hline Central & 23,4 & $22,4-24,4$ & 12,0 & $8,78-16,3$ & 16,3 & $12,9-20,3$ & 25,0 & $23,9-26,2$ \\
\hline Pacífica & 15,8 & $15,0-16,5$ & 34,4 & $28,7-40,7$ & 42,9 & $38,8-47,1$ & 10,9 & $10,1-11,7$ \\
\hline Bogotá & 17,2 & $16,2-18,2$ & 3,4 & $1,8-6,7$ & 1,6 & $0,8-3,2$ & 20,1 & $19,0-21,3$ \\
\hline Orinoquía y Amazonía & 2,8 & $2,6-3,0$ & 12,0 & $10,2-14,1$ & 0,6 & $0,4-1,0$ & 2,6 & $2,4-2,8$ \\
\hline \multicolumn{9}{|l|}{ Zona de residencia ** } \\
\hline Urbano & 73,7 & $72,7-74,8$ & 40,4 & $35,1-46,0$ & 70,3 & $66,5-73,9$ & 76,1 & $74,9-77,2$ \\
\hline Rural & 26,3 & $25,3-27,3$ & 59,6 & $54,0-64,9$ & 29,7 & $26,1-33,5$ & 23,9 & $22,8-25,1$ \\
\hline
\end{tabular}

ES: error estándar; IC95\%: intervalo del 95\% de confianza.

* Para variables continuas se presenta la media y el error estándar y en las variables categóricas porcentajes e IC95\%;

$\star \star$ Valor de $\mathrm{p}<0,01$.

$\star \star \star$ Valor de $p<0,05$ 
to educativo, laboral y económico. Las mujeres indígenas presentaron los menores niveles de escolaridad (6 años de estudio en promedio), el mayor porcentaje sin educación (10,4\%), el menor porcentaje en educación superior $(9,7 \%)$, la menor proporción de hogares con jefatura femenina (24\%), y el mayor porcentaje sin trabajo $(19,9 \%)$. Con respecto a la afiliación al sistema general de seguridad social en salud, alrededor el $9,6 \%$ no se encontraba afiliada. Por último, $78 \%$ de los hogares de las mujeres indígenas se encontraba en los menores niveles socioeconómicos (bajo y más bajo).

\section{Inequidades en la utilización de servicios de control prenatal, atención del parto y posparto}

El 97\% de las mujeres incluidas en el estudio se realizó al menos un control prenatal. Al comparar entre los grupos el porcentaje fue menor en las mujeres indígenas (90,8\%). Analizando el control prenatal de acuerdo al índice de Kotelchuck encontramos que en los tres grupos el porcentaje de atención "inadecuado" fue superior al $20 \%$, llegando a 39,9\% en las indígenas. El control prenatal calificado como intermedio fue mayor en las mujeres sin pertenencia étnica $(77,9 \%)$. Sólo 2,1\% de las mujeres tuvo un número adecuado de controles prenatales, siendo mayor en las afrodescendientes (2,6\%) que en los otros dos grupos (Tabla 2).

La atención del parto por profesionales de la salud se presentó en el 97,4\%, pero fue menor en la población indígena $(86,9 \%)$, la atención por partera fue mayor en la población indígena $(12,2 \%)$ y ocurrió en $5,4 \%$ de las afrodescendientes. El 1\% de las indígenas reportó que nadie atendió su parto. Referente a la utilización de los servicios de salud para la atención del parto, fue de $95,5 \%$, con un menor porcentaje en las indígenas $(75,7 \%)$. Al indagar sobre los motivos de la no atención institucional del parto, los más frecuentes fueron la lejanía $(38,3 \%)$; los costos del transporte (19\%); y la desconfianza en los médi$\cos (8,2 \%)$ (Tabla 2$)$.

Referente al control posparto, fue realizado en $74,9 \%$ de las mujeres. Las indígenas y afrodescendientes presentaron los menores porcentajes (54\% y $66 \%$ respectivamente). En las mujeres que no pertenecen a ninguna etnia, el porcentaje de utilización fue superior al promedio general $(77,3 \%)$ (Tabla 2).
Regresión logística ordinal para

\section{el índice de Kotelchuck}

El modelo de la Tabla 3 muestra una asociación inversa entre la etnia y la atención prenatal: indígenas, (OR = 0,61; IC95\%: 0,50-0,74); afrodescendientes, $(\mathrm{OR}=0,79$; IC95\%: 0,67-0,94). De igual manera, en las mujeres con familia extensa, compuesta y con antecedente de mortalidad infantil, disminuyen las posibilidades de control prenatal. Por otro lado, se encontró una asociación directa entre estar afiliado a la seguridad social y tener mayores controles prenatales; siendo mayor la posibilidad en el régimen contributivo, (OR = 1,88; IC95\%: 1,54-2,29) y subsidiado (OR = 1,40; IC95\%: 1,19-1,69) que en las no afiliadas. En las mujeres hospitalizadas durante el embarazo fueron mayores las posibilidades de mejor control prenatal. En este modelo, los puntos de corte $(c u t)$ indican el incremento proporcional seguido en las variables latentes.

\section{Regresión logística binomial para la atención del parto y posparto}

En la Tabla 4, se presenta la asociación inversa entre la etnia y la atención institucional del parto; indígenas, (OR = 0,33; IC95\%: 0,25-0,44); afrodescendientes, $(\mathrm{OR}=0,60$; IC95\%: 0,44-0,80). Entre las mujeres que trabajan en la agricultura son menores las posibilidades de atención del parto en los servicios de salud (OR = 0,61; IC95\%: 0,440,86). Por otro lado, se encontró una asociación directa entre el control prenatal y la atención institucional del parto: intermedio, $(\mathrm{OR}=2,70$; IC95\%: 2,19-3,33); adecuado/plus, $(\mathrm{OR}=4,33$; IC95\%: 1,61-11,68). Las mujeres que se encontraban en el área urbana, y, afiliadas al sistema de salud también presentaron mayores posibilidades de atención del parto en los servicios de salud. Otros factores asociados con la atención del parto se encuentran en la Tabla 4 .

Con respecto al posparto, también se encontró una asociación inversa con la etnia. Siendo menores las posibilidades de recibir este servicio para las mujeres indígenas, $(\mathrm{OR}=0,80$; IC95\%: 0,65-0,98); y afrodescendientes $(\mathrm{OR}=0,80$; IC95\%: 0,68-0,94). En contraste, se evidenció una asociación directa entre la atención institucional del parto y el control posparto $(\mathrm{OR}=2,79$; IC95\%: 2,24-3,48). Las mujeres que recibieron atención prenatal, y las que fueron hospitalizadas durante el embarazo presentaron mayores posibilidades de una atención posparto (Tabla 4). 
Características de la utilización de los servicios de salud reproductiva según la etnia.

\begin{tabular}{|c|c|c|c|c|c|c|c|c|}
\hline & \multicolumn{2}{|c|}{$\begin{array}{c}\text { Total } \\
(\mathrm{N}=14.514)\end{array}$} & \multicolumn{2}{|c|}{$\begin{array}{l}\text { Indigena } \\
(n=1.962)\end{array}$} & \multicolumn{2}{|c|}{$\begin{array}{l}\text { Afrodescendete } \\
\qquad(n=1.729)\end{array}$} & \multicolumn{2}{|c|}{$\begin{array}{c}\text { Ninguna } \\
(\mathrm{n}=10.823)\end{array}$} \\
\hline & $\%$ & IC95\% & $\%$ & IC95\% & $\%$ & IC95\% & $\%$ & $1 \mathrm{C} 95 \%$ \\
\hline \multicolumn{9}{|l|}{ Al menos un control prenatal * } \\
\hline Sí & 97,0 & $96,6-97,3$ & 90,8 & $88,2-92,8$ & 95,7 & $94,5-96,7$ & 97,5 & $97,1-97,9$ \\
\hline \multicolumn{9}{|l|}{ Índice de Kotelchuck de controles } \\
\hline \multicolumn{9}{|l|}{ prenatales * } \\
\hline Inadecuado & 21,7 & $20,8-22,7$ & 39,9 & $35,9-44,1$ & 26,6 & $23,9-29,4$ & 20,0 & $19,0-21,0$ \\
\hline Intermedio & 76,2 & $75,2-77,2$ & 59,0 & $54,9-63,0$ & 70,9 & $68,0-73,9$ & 77,9 & $76,9-78,9$ \\
\hline Adecuado o adecuado plus & 2,1 & $1,8-2,5$ & 1,1 & $0,5-2,3$ & 2,6 & $1,8-3,7$ & 2,1 & $1,8-2,5$ \\
\hline \multicolumn{9}{|l|}{ Personal que atendió el parto * } \\
\hline Profesional de la salud & 97,4 & $97,1-97,8$ & 86,9 & $82,6-90,2$ & 94,3 & $92,5-95,7$ & 98,4 & $98,1-98,7$ \\
\hline Partera & 2,3 & $2,0-2,7$ & 12,2 & $8,9-16,4$ & 5,4 & $4,1-7,2$ & 1,4 & $1,2-1,7$ \\
\hline Nadie & 0,2 & $0,2-0,3$ & 1,0 & $0,5-1,9$ & 0,3 & $0,1-0,7$ & 0,2 & $0,1-0,3$ \\
\hline \multicolumn{9}{|l|}{ Atención del parto en los servicios } \\
\hline \multicolumn{9}{|l|}{ de salud * } \\
\hline Sí & 95,5 & $95,0-96,0$ & 75,7 & $70,9-80,0$ & 91,6 & $89,4-93,3$ & 97,2 & $96,7-97,5$ \\
\hline \multicolumn{9}{|c|}{ Motivos de no atención del parto en } \\
\hline \multicolumn{9}{|c|}{ los servicios de salud $(n=1,261)$} \\
\hline \multicolumn{9}{|l|}{ El servicio queda lejos * } \\
\hline Sí & 38,3 & $34,1-42,6$ & 31,4 & $25,1-38,6$ & 48,2 & $39,5-56,9$ & 37,4 & $31,4-43,8$ \\
\hline \multicolumn{9}{|l|}{ El transporte es muy costoso * } \\
\hline Sí & 19,0 & $15,8-22,6$ & 16,6 & $11,4-23,5$ & 29,6 & $21,6-39,0$ & 15,5 & $11,6-20,3$ \\
\hline \multicolumn{9}{|l|}{ No confía en los médicos ** } \\
\hline Sí & 8,2 & $6,1-11,0$ & 5,0 & $3,1-8,0$ & 7,0 & $3,8-12,8$ & 10,4 & $6,9-15,3$ \\
\hline \multicolumn{9}{|l|}{ Control posparto * } \\
\hline Sí & 74,9 & $73,9-75,9$ & 54,2 & $50,0-58,3$ & 66,0 & $63,0-69,0$ & 77,3 & $76,2-78,3$ \\
\hline
\end{tabular}

IC95\%: intervalo del 95\% de confianza.

* Valor de $\mathrm{p}<0,01$;

** Valor de $p>0,05$

\section{Discusión}

En este estudio encontramos una mayor proporción de mujeres indígenas y afrodescendientes sin atención prenatal o con una inadecuada atención, con menor atención del parto por profesionales, con menor utilización de servicios de salud para la atención del parto, y del posparto, que llega en el caso de las indígenas sólo al 54\%. Esto refleja inequidades en salud, considerando la alta cobertura de estos servicios en las mujeres de ninguna etnia, lo cual evidencia que en Colombia los derechos reproductivos no están siendo garantizados en todas las mujeres. Estos resultados coinciden con otros estudios realizados que evidencian obstáculos administrativos, geográficos, económicos y culturales 17,22,27,38,39. Sin embargo, es necesario continuar incrementando la evidencia científica sobre el acceso a los servicios de salud reproductiva que viven las mujeres de los grupos étnicos del país.
Es probable que las mujeres con controles prenatales inadecuados, no hayan recibido una atención prenatal como lo recomiendan las guías de práctica clínica, situación preocupante, debido a la importancia que tiene el control prenatal para la salud del binomio madre-hijo 21 . El observatorio de salud materna de Colombia reporta que desde el 2005 hasta el 2010 la totalidad de los partos en el país han sido atendidos por personal cualificado en los servicios de salud 40 . Nuestros resultados difieren de dicha cifra, y evidencian la vigencia de las parteras, quienes contribuyen a garantizar la atención de las gestantes principalmente de las comunidades étnicas, durante la gestación, el parto y el puerperio 41,42 .

Las barreras geográficas, económicas y la poca confianza en los médicos, que han sido reportadas en otros estudios del país 11,17,25,26, continúan siendo los principales motivos para no utilizar los servicios de salud reproductiva. Esta situación refleja que el aseguramiento en 
Modelo de regresión logística ordinal para el índice de Kotelchuck *

\begin{tabular}{|c|c|c|}
\hline Variable & OR & IC95\% \\
\hline \multicolumn{3}{|l|}{ Etnicidad (ref. ninguna) } \\
\hline Indígena & 0,61 & $0,50-0,74$ \\
\hline Afrodescendiente & 0,79 & $0,67-0,94$ \\
\hline \multicolumn{3}{|c|}{ Región (ref. Orinoquía y Amazonía) } \\
\hline Atlántica & 1,51 & $1,29-1,76$ \\
\hline Oriental & 1,05 & $0,89-1,25$ \\
\hline Central & 1,46 & $1,23-1,74$ \\
\hline Pacífica & 1,57 & $1,32-1,80$ \\
\hline Bogotá & 1,11 & $0,87-1,42$ \\
\hline \multicolumn{3}{|l|}{ Zona de residencia (ref. rural) } \\
\hline Urbano & 1,10 & $0,94-1,29$ \\
\hline \multicolumn{3}{|c|}{ Índice de riqueza hogar (ref. más bajo) } \\
\hline Bajo & 1,57 & $1,34-1,85$ \\
\hline Medio & 1,53 & $1,25-1,87$ \\
\hline Alto & 1,99 & $1,56-2,54$ \\
\hline Más alto & 1,88 & $1,43-2,48$ \\
\hline \multicolumn{3}{|c|}{ Afiliación en salud (ref. no afiliada) } \\
\hline Contributivo & 1,88 & $1,54-2,29$ \\
\hline Subsidiado & 1,40 & $1,19-1,64$ \\
\hline \multicolumn{3}{|l|}{ Tipo de familia (ref. nuclear) } \\
\hline Extensa & 0,80 & $0,72-0,90$ \\
\hline Compuesta & 0,70 & $0,55-0,88$ \\
\hline \multicolumn{3}{|l|}{ Jefatura hogar (ref. mujer) } \\
\hline Hombre & 1,19 & $1,05-1,34$ \\
\hline Años de escolaridad ** & 1,09 & $1,07-1,11$ \\
\hline \multicolumn{3}{|c|}{ Ocupación laboral (ref. no trabaja) } \\
\hline Profesional/técnico & 1,02 & $1,01-1,03$ \\
\hline Oficinista & 1,03 & $0,761,38$ \\
\hline Ventas y servicios & 0,92 & $0,70-1,21$ \\
\hline Agricultura & 1,01 & $0,86-1,19$ \\
\hline Manual & 1,00 & $0,78-1,27$ \\
\hline Edad (años) ** & 1,07 & $0,81-1,40$ \\
\hline \multicolumn{3}{|c|}{ Hospitalización durante embarazo (ref. no) } \\
\hline Sí & 1,32 & $1,15-1,50$ \\
\hline \multicolumn{3}{|c|}{ Antecedente de mortalidad infantil (ref. no) } \\
\hline Sí & 0,75 & $0,61-0,91$ \\
\hline Cut 1 & 1,16 & $0,80-1,52$ \\
\hline Cut 2 & 6,66 & $6,22-7,10$ \\
\hline
\end{tabular}

IC95\%: intervalo del 95\% de confianza; OR: odds ratio, razón de momios.

* Modelo de regresión logística ordinal, Variable dependiente: índice de Kotelchuck en escala ordinal (altos resultados indican mejor control prenatal);

** Variables continuas.

Colombia no es suficiente para el lograr el acceso de las poblaciones más dispersas y con bajos recursos económicos 25 , y que a través de este modelo de mercado solo se logra una relación contractual que resulta insuficiente para garantizar el derecho a la salud 43. Es necesaria una relación más igualitaria entre usuarias y prestadores, basada en un objetivo común, que permita a las usuarias identificar los servicios de salud como el lugar para satisfacer sus necesidades en salud, basados en la confianza 44. Acerca de la atención posparto, otros estudios igualmente 
Modelos de regresión logística binomial para la atención del parto y posparto en los servicios de salud.

\begin{tabular}{|c|c|c|c|c|}
\hline & \multicolumn{2}{|c|}{ Atención del parto } & \multicolumn{2}{|c|}{ Atención posparto } \\
\hline & OR & IC95\% & OR & IC95\% \\
\hline \multicolumn{5}{|l|}{ Etnia (ref. ninguna) } \\
\hline Indígena & 0,33 & $0,25-0,44$ & 0,80 & $0,65-0,98$ \\
\hline Afrodescendiente & 0,60 & $0,44-0,80$ & 0,80 & $0,68-0,94$ \\
\hline \multicolumn{5}{|c|}{ Región (ref. Orinoquía y Amazonía) } \\
\hline Atlántica & 2,18 & $1,56-3,04$ & 0,53 & $0,45-0,63$ \\
\hline Oriental & 2,26 & $1,57-3,26$ & 0,86 & $0,71-1,04$ \\
\hline Central & 1,88 & $1,32-2,69$ & 1,71 & $1,40-2,09$ \\
\hline Pacífica & 1,12 & $0,80-1,55$ & 1,09 & $0,90-1,32$ \\
\hline Bogotá & 2,90 & $1,10-7,67$ & 1,26 & $0,98-1,64$ \\
\hline \multicolumn{5}{|c|}{ Zona de residencia (ref. rural) } \\
\hline Urbano & 1,94 & $1,44-2,63$ & 0,96 & $0,81-1,13$ \\
\hline \multicolumn{5}{|c|}{ Índice de riqueza hogar (ref. más bajo) } \\
\hline Bajo & 1,51 & $1,12-2,03$ & 1,19 & $1,02-1,38$ \\
\hline Medio & 4,17 & $2,27-7,67$ & 1,29 & $1,05-1,57$ \\
\hline Alto & 2,75 & $1,26-5,98$ & 1,89 & $1,48-2,40$ \\
\hline Más alto & 0,99 & $0,48-2,07$ & 2,22 & $1,61-3,06$ \\
\hline \multicolumn{5}{|c|}{ Afiliación en salud (ref. no afiliada) } \\
\hline Contributivo & 2,76 & $1,57-4,86$ & 2,11 & $1,75-2,56$ \\
\hline Subsidiado & 1,48 & $1,14-1,92$ & 1,29 & $1,11-1,50$ \\
\hline \multicolumn{5}{|c|}{ Tipo de familia (ref. nuclear) } \\
\hline Extensa & 1,30 & $1,05-1,62$ & 0,97 & $0,86-1,08$ \\
\hline Compuesta & 0,95 & $0,60-1,51$ & 0,82 & $0,64-1,05$ \\
\hline \multicolumn{5}{|c|}{ Jefatura hogar (ref. mujer) } \\
\hline Hombre & 1,19 & $0,92-1,54$ & 1,02 & $0,90-1,16$ \\
\hline Escolaridad (años) * & 1,16 & $1,12-1,20$ & 1,07 & $1,05-1,09$ \\
\hline \multicolumn{5}{|c|}{ Ocupación laboral (ref. no trabaja) } \\
\hline Profesional/técnico & 1,57 & $0,35-6,97$ & 1,19 & $0,85-1,66$ \\
\hline Oficinista & 1,12 & $0,49-2,55$ & 1,60 & $1,16-2,21$ \\
\hline Ventas y servicios & 0,90 & $0,68-1,19$ & 0,95 & $0,82-1,09$ \\
\hline Agricultura & 0,61 & $0,44-0,86$ & 1,26 & $0,98-1,62$ \\
\hline Manual & 1,08 & $0,60-1,96$ & 0,89 & $0,67-1,18$ \\
\hline Edad (años) * & 1,00 & $0,98-1,01$ & 1,02 & $1,01-1,03$ \\
\hline \multicolumn{5}{|c|}{ Control prenatal (ref. inadecuado) } \\
\hline Intermedio & 2,70 & $2,19-3,33$ & 1,76 & $1,57-1,99$ \\
\hline Adecuado/Plus & 4,33 & $1,61-11,68$ & 2,59 & $1,61-4,15$ \\
\hline \multicolumn{5}{|c|}{ Hospitalización durante embarazo (ref. no) } \\
\hline Sí & 2,89 & $1,96-4,25$ & 1,25 & $1,09-1,43$ \\
\hline \multicolumn{5}{|c|}{ Antecedente de mortalidad infantil (ref. no) } \\
\hline $\mathrm{Si}$ & 0,76 & $0,55-1,05$ & 0,91 & $0,73-1,13$ \\
\hline \multicolumn{5}{|c|}{ Atención institucional del parto (ref. no) } \\
\hline $\mathrm{Si}$ & - & - & 2,79 & $2,24-3,48$ \\
\hline
\end{tabular}

IC95\%: intervalo del 95\% de confianza; OR: odds ratio, razón de momios.

* Variables continuas.

demuestran una baja prevalencia en el uso de este servicio, al cual se le resta importancia o solo se acude en caso de alguna complicación obstétrica 45,46 .
Otro elemento identificado como marcador de inequidad es la poca continuidad, puesto que la diferencia entre el porcentaje de mujeres que inician el control prenatal y las que al final reciben atención posparto es importante: $36 \%$ en 
indígenas, $30 \%$ en afrodescendientes, y, 20\% en mujeres que no forman parte de ninguna etnia. La asociación del control prenatal con la atención institucional del parto y del posparto también ha sido reportada en otros estudios $46,47,48$, lo cual permite reafirmar que el control prenatal es el punto de partida para la continuidad en la atención. Allí se identifican riesgos del embarazo, y se genera la confianza necesaria entre la paciente, su familia y los servicios de salud, que influye en una demanda posterior de los servicios de salud 7 .

Analizar las inequidades encontradas únicamente desde el punto de vista de la etnia es limitado y puede estar sesgado 32 , dado que la situación de las mujeres indígenas y afrodescendientes del país están marcadas por una triple inequidad: de etnia, de género y clase social. Son mujeres indígenas y afrodescendientes las que presentan menores niveles educativos, mayor desempleo y menor acceso a trabajos calificados y hogares con los niveles socioeconómicos más bajos. Igualmente, las indígenas por razones culturales tienen menor empoderamiento para la toma de decisiones sobre su salud y la de su familia, lo cual puede explicar que aquellas que viven con familia extensa tengan menor posibilidad de tener el número adecuado y oportuno de controles prenatales 49,50. Esta triple carga de inequidad también está relacionada con el contexto político y social marcado por un escenario de violencia y conflicto armado interno, el cual ha generado el desplazamiento masivo de poblaciones al interior de la región o hacia las principales ciudades del país 51,52. Según el Alto Comisionado de las Naciones Unidas para los Refugiados (ACNUR) 53, aproximadamente el $2 \%$ del total de personas desplazadas del país pertenece a alguna etnia indígena y miles de afrodescendientes han migrado a las ciudades y sobreviven en condiciones de extrema pobreza, con condiciones laborales precarias.

Otro aspecto a tener en cuenta hace referencia a la regionalización de la inequidad, y lo que Krieger 53 denomina discriminación institucional a nivel poblacional. Gran proporción de los grupos indígenas y afrodescendientes del país reside en la región pacífica, zona que presenta en su mayoría los indicadores socioeconómicos y de salud más desfavorables del país, y por su diversidad geográfica es unas de los territorios del país con mayores dificultades de acceso terrestre, con limitaciones de acceso a servicios públicos, y menor empoderamiento político de sus mujeres 17,55 . Todos estos elementos se constituyen en factores que ahondan la problemática de inequidad social y de salud presente en las mujeres indígenas y afrodescendientes.
Los resultados expuestos deben ser interpretados con cautela considerando, que el autoreporte puede implicar subregistro. Por último, es importante considerar que las disparidades encontradas en las variables sociales y de salud entre los grupos no pueden ser atribuidas únicamente a la etnia de las mujeres, ni sacadas del contexto dónde emergen, puesto que envuelven aspectos históricos, políticos y socioculturales.

\section{Conclusión}

El panorama encontrado muestra la existencia de inequidades sociales y de salud reproductiva en las mujeres de las minorías étnicas, brechas que representan una deuda histórica con los pueblos indígenas y afrodescendientes, y deben cerrarse con estrategias gubernamentales que garanticen el derecho a la salud reconociendo la diversidad, y respetando la autonomía de los pueblos indígenas plasmadas en la Declaración de las Naciones Unidas sobre los derechos de los pueblos indígenas de 2007 56. Lo anterior, considerando que Colombia tiene un bloque constitucional que reconoce la existencia y los derechos de las comunidades étnicas 57 , que define la salud como derecho fundamental en un marco de principios de equidad e interculturalidad (Ley Estatutaria 1.751 de 2015 58), y que cuenta con políticas públicas para garantizar la equidad de género, los derechos de los grupos étnicos y los derechos sexuales y derechos reproductivos de la población 59 .

El presente estudio contribuye a visibilizar la situación de acceso a servicios de salud de grupos étnicos que han vivido históricamente en condiciones de vulneración de sus derechos, lo que constituye un aporte a la generación de evidencia científica de las inequidades en salud de las mujeres pertenecientes a las etnias de Colombia, a partir del uso de información con representatividad nacional disponible y poco utilizada, que puede convertirse en una herramienta técnica para sustentar la toma de decisiones, con el fin de garantizar el acceso a los servicios de salud reproductiva en un marco de equidad. Para lograrlo se hace necesario atender las condiciones de exclusión social de la que son objeto, con perspectivas multiculturales y modelos de atención en salud diferenciados, según la etnia y la región, sobre los cuales existen experiencias en Colombia y en América Latina 22,41,60, que permita superar las barreras existentes para la atención de la salud reproductiva de las mujeres del país, como un deber ético y de justicia social del Estado. 


\section{Resumo}

O objetivo deste estudo na Colômbia foi analisar o uso de serviços de saúde reprodutiva (pré-natal, parto e pós-parto) de acordo com a identidade étnica das mulheres, com base na Pesquisa Nacional sobre Demografia e Saúde de 2010. A identidade étnica era autorreferida (indígena, afrodescendente ou nenhuma das duas), e modelos logísticos foram aplicados a cada serviço de saúde. As mulheres colombianas indígenas e afrodescendentes usavam menos os serviços de saúde reprodutiva, quando comparadas àquelas que não informaram a identidade étnica. De acordo com os modelos de regressão, as mulheres indígenas mostravam menores chances de ter um número adequado de consultas de pré-natal $(O R=0,61)$, o mesmo valendo para as afrodescendentes. As mesmas disparidades apareciam em relação ao parto hospitalar: parturientes indigenas $(O R=0,33)$ e afrodescendentes $(O R=0,60)$; e atendimento puerperal: indígenas $(O R=0,80)$ e afrodescendentes $(\mathrm{OR}=0,80)$. O estudo concluiu que há desigualdades no uso de serviços de saúde reprodutiva por mulheres pertencentes a grupos étnicos na Colômbia, e que estas devem ser alvo de estratégias públicas para garantir seu direito à saúde.

Desigualdades em Saúde; Serviços de Saúde Reprodutiva; Grupos Étnicos; Direitos Reprodutivos

\section{Colaboradores}

C. Noreña-Herrera participó en la concepción del estudio y en todas las etapas de la producción de este artículo y fue el responsable de elaborar la versión de este artículo para ser sometida a publicación. R. Leyva-Flores participó en la concepción del estudio, la redacción del artículo y revisión crítica del contenido intelectual, así como la aprobación de la versión final. L. S. PalacioMejía participó en el análisis e interpretación de los datos, en la redacción del artículo, y en la aprobación de la versión final a ser publicada. M. B. Duarte-Gómez participó revisión crítica del contenido intelectual, redacción del artículo y la aprobación de la versión final a publicarse.

\section{Agradecimientos}

A los profesores del Colegio de Salud Reproductiva del Instituto Nacional de Salud Pública de México por su apoyo académico y científico, así como a los evaluadores de este artículo, por sus acertados comentarios y recomendaciones. C. Noreña-Herrera agradece la financiación recibida del Consejo Nacional de Ciencia y Tecnología de México (CONACYT).

\section{Referencias}

1. World Health Organization. Universal access to reproductive health: accelerated actions to enhance progress on Millennium Development Goal 5 through advancing Target 5B. Geneva: World Health Organization; 2011.

2. Naciones Unidas. Objetivos de desarrollo del milenio: informe de 2014. New York: Naciones Unidas; 2014.

3. Organización Panamerica de la Salud/Organización Mundial de la Salud. Situación de salud en las Américas: indicadores básicos 2014. Washington DC: Organización Panamerica de la Salud/Organización Mundial de la Salud; 2014.

4. Departamento Nacional de Planeación. Metas y estrategias de Colombia para el logro de los Objetivos de Desarrollo del Milenio-2015. CONPES Social 91. Bogotá DC: Departamento Nacional de Planeación; 2011. (Documentos Técnicos y de Política).

5. World Health Organization. WHO antenatal care randomized trial. Manual for the implementation of the new model. Geneva: World Health Organization; 2003.

6. Freyermuth G, Sesia P. La muerte materna: acciones y estrategias hacia una maternidad segura. México DF: Centro de Investigaciones y Estudios Superiores en Antropología Social; 2009.

7. World Health Organization. WHO technical consultation on postpartum and postnatal care. Geneva: World Health Organization; 2010.

8. Comisión sobre Determinantes Sociales de la Salud, Organización Mundial de la Salud. Subsanar las desigualdades en una generación: alcanzar la equidad sanitaria actuando sobre los determinantes sociales de la salud. http://whqlibdoc.who.int/ hq/2008/WHO_IER_CSDH_08.1_spa.pdf (accedido el 20/Feb/2010).

9. Balladeli P-P, Guzmán J-M, Korc M, Moreno P, Rivera G. Equidad en salud para las minorías étnicas en Colombia. Experiencias de acción intersectorial en Colombia para la equidad en salud de las minorías étnicas. Bogotá DC: Organización Panamericana de la Salud; 2013.

10. Torres C. Equity in health as seen from an ethnic focus. Rev Panam Salud Pública 2001; 10:188-201.

11. Ishida K, Stupp P, Turcios-Ruiz R, William DB, Espinoza E. Ethnic inequality in Guatemalan women's use of modern reproductive health care. Int Perspect Sex Reprod Health 2012; 38:99-108.

12. Calfio M, Velasco L. Mujeres indígenas en América Latina: brechas de género o de étnia? Santiago de Chile: Comisión Económica para América Latina y el Caribe; 2005.

13. Rodríguez L. Factores sociales y culturales determinantes en salud: la cultura como una fuerza para incidir en cambios en políticas de salud sexual y reproductiva. Quito: Fondo de Población de las Naciones Unidas; 2008.

14. Oyarce AM, Ribotta B, Pedrero M. Salud materno infantil de pueblos indígenas y afrodescendientes de América Latina: aportes para una relectura desde el derecho a la integridad cultural. Santiago de Chile: Naciones Unidas; 2010. 
15. Programa de las Naciones Unidas para el Desarrollo. Pueblos indígenas y los objetivos de desarrollo del milenio. Bogotá DC: Programa de las Naciones Unidas para el Desarrollo; 2013.

16. Acosta Y, Segura O. Indígenas gestantes en la ribera del Amazonas, Colombia, 2009: conocimientos, actitudes y prácticas. Investig Andina 2011; 13:108-20.

17. Martínez F, Castro C, Ordóñez I, Valencia O, Hinestroza Y. El sistema de seguridad social en salud en la región Pacifica Colombiana: la problemática de la población dispersa. Bogotá DC: Fundación para la Investigación y el Desarrollo de la Salud y la Seguridad Social; 2005.

18. Departamento Administrativo Nacional de Estadística. Censo general 2005: nivel nacional. Bogotá: Departamento Administrativo Nacional de Estadística; 2008.

19. Departamento Administrativo Nacional de Estadística. Colombia una nación multicultural: su diversidad étnica. Bogotá: Departamento Administrativo Nacional de Estadística; 2007.

20. Congreso de la República de Colombia. Ley 100 de 1993 por la cual se crea el sistema de seguridad social integral y se dictan otras disposiciones. Diario Oficial 41148; 23 dic.

21. Ministerio de Salud y Protección Social. Guías de práctica clínica para la prevención, detección temprana y tratamiento del embarazo, parto o puerperio. Bogotá: Ministerio de Salud y Protección Social; 2013.

22. Duarte-Gómez M, Gómez Dantés O, Alegría O, Liliana M, Ríos M, Hernando L, et al. Un modelo de atención en salud al pueblo Wayúu en la frontera colombo-venezolana. Rev Panam Salud Pública $2011 ; 30: 272-8$.

23. Fondo de las Naciones Unidas para la Infancia. Los pueblos indígenas en Colombia. Derechos, políticas y desafíos. Bogotá: Fondo de las Naciones Unidas para la Infancia; 2003.

24. Temmerman M, Khosla R, Say L. Sexual and reproductive health and rights: a global development, health, and human rights priority. Lancet 2014; 384:e30-1.

25. Vargas-Jaramillo J, Molina-Marín G. Acceso a los servicios de salud en seis ciudades de Colombia: limitaciones y consecuencias. Rev Fac Nac Salud Pública 2009; 27:121-30.

26. Ariza-Montoya JF, Hernández-Álvarez ME. Equidad de etnia en el acceso a los servicios de salud en Bogotá, Colombia, 2007. Rev Salud Pública 2008; 10 Suppl 1:58-71.

27. Osorio AM, Tovar LM, Rathmann K. Individual and local level factors and antenatal care use in Colombia: a multilevel analysis. Cad Saúde Pública 2014; 30:1079-92.

28. Asociación Probienestar de la Familia Colombiana. Salud sexual y reproductiva en Colombia: encuesta nacional de demografía y salud 2005. Bogotá: Asociación Probienestar de la Familia Colombiana; 2005.

29. Wade P, Beltrán CLL, Restrepo E, Santos RV. Mestizo genomics: race mixture, nation, and science in Latin America. Durham: Duke University Press; 2014.
30. Ford CL, Airhihenbuwa CO. Critical race theory, race equity, and public health: toward antiracism praxis. Am J Public Health 2010; 100 Suppl 1:S30-5.

31. Kabad JF, Bastos JL, Santos RV. Race, color and ethnicity in epidemiologic studies carried out with Brazilian populations: systematic review on the PubMed database. Physis (Rio J.) 2012; 22:895-918.

32. Vanegas LJ, Villalón CM, Valenzuela YC. Consideraciones acerca del uso de la variable etnia/raza en investigación epidemiológica para la Salud Pública: a propósito de investigaciones en inequidades. Rev Médica Chile 2008; 136:637-44.

33. del Popolo F. Los pueblos indígenas y afrodescendientes en las fuentes de datos: experiencias en América Latina. Santiago de Chile: Organización Panamericana de la Salud; 2008.

34. Asociación Probienestar de la Familia Colombiana. Colombia: Encuesta Nacional de Demografía y Salud, 2010. Bogotá: Asociación Probienestar de la Familia Colombiana; 2011.

35. Kotelchuck M. An evaluation of the Kessner adequacy of prenatal care index and a proposed adequacy of prenatal care utilization index. Am J Public Health 1994; 84:1414-20.

36. Filmer D, Pritchett L. Estimating wealth effects without expenditure data or tears: an application to educational enrollments in states of India. Demography 2001; 38:115-32.

37. Hosmer DWH, Lemeshow S, Sturdivant RX. Applied logistic regression. New York: John Wiley \& Sons; 2013.

38. Asociación Probienestar de la Familia Colombiana. Encuesta en zonas marginadas: Salud sexual y reproductiva, desplazamiento forzado y pobreza 2000-2011. Bogotá: Asociación Probienestar de la Familia Colombiana; 2011.

39. Ariza-Montoya JF, Hernández-Álvarez ME. Ethnic equity in accessing health services in Bogotá, Colombia, 2007. Rev Salud Pública 2008; 10:58-71.

40. Ministerio de la Salud y Protección Social, Sistema Integral de Información de la Protección Social. Observatorio de Salud Materna de Colombia. http://www.sispro.gov.co/Pages/Observatorios/ salud_materna.aspx (accedido el 09/May/2015).

41. Fondo de Población de las Naciones Unidas. El estado de las parteras en el mundo 2014 : hacia el acceso universal a la salud, un derecho de la mujer. New York: Naciones Unidas; 2014.

42. Hincapié E, Valencia CP. Capacitación de las parteras y su relación con la mortalidad perinatal del municipio de Quinchía, Colombia. Colomb Méd 2000; 31:11-5.

43. Álvarez MH. El derecho a la salud en Colombia: obstáculos estructurales para su realización. Rev Salud Pública 2010; 2:121-44.

44. Trivedi P, Wykes T. From passive subjects to equal partners. Br J Psychiatry 2002; 181:468-72.

45. Titaley CR, Dibley MJ, Roberts CL. Factors associated with non-utilisation of postnatal care services in Indonesia. J Epidemiol Community Health 2009; 63:827-31.

46. Regassa N. Antenatal and postnatal care service utilization in southern Ethiopia: a populationbased study. Afr Health Sci 2011; 11:390-7. 
47. Khanal V, Adhikari M, Karkee R, Gavidia T. Factors associated with the utilisation of postnatal care services among the mothers of Nepal: analysis of Nepal demographic and health survey 2011. BMC Womens Health 2014; 14:19.

48. Feinstein L, Dimomfu BL, Mupenda B, Duvall S, Chalachala JL, Edmonds A, et al. Antenatal and delivery services in Kinshasa, Democratic Republic of Congo: care-seeking and experiences reported by women in a household-based survey. Trop Med Int Health 2013; 18:1211-21.

49. Espinosa G. Doscientas trece voces contra la muerte: mortalidad materna en zonas indígenas. In: Castañeda M, Díaz D, Espinosa G, Freyermuth G, Sanchez D, editores. La mortalidad materna en México: cuatro visiones críticas. México DF: Universidad Autónoma Metropolitana; 2004. p. 161-238.

50. Valdez-Santiago R, Pelcastre-Villafuerte B, DuarteGómez MB, Cuadra-Hernández SM, Yinhué MS. De lo local a lo global: políticas públicas para el empoderamiento de las mujeres indígenas Evaluación del acuerdo de colaboración CDI-ONU Mujeres, en el marco del Programa de Acciones para la Igualdad de Género con Pueblos Indígenas. México DF: Instituto Nacional de Salud Pública; 2013.

51. Bello M. El desplazamiento forzado en Colombia: acumulación de capital y exclusión social. Aportes Andinos 2003; 7:1-8.

52. Egea Jiménez C, Suescún S, Iván J. Migraciones y conflictos: el desplazamiento interno en Colombia. Convergencia 2008; 15:207-35.
53. Agencia de la ONU para los Refugiados. Desplazamiento interno en Colombia. http://www.acnur. org/t3/operaciones/situacion-colombia/despla zamiento-interno-en-colombia/ (accedido el 09/ May/2015).

54. Krieger N. Discrimination and health inequities. Social epidemiology. New York: Oxford University Press; 2014.

55. Comisión Económica para America Latina y el Caribe; Fundación Ford. Atlas sociodemográfico de los pueblos indígenas y afrodescendientes en Colombia. Santiago de Chile: Naciones Unidas; 2012.

56. Naciones Unidas. Declaración de las Naciones Unidas sobre los derechos de los pueblos indígenas. s.l.: Naciones Unidas; 2007. (Report 61/295).

57. Asamblea Nacional Constituyente. Constitución Política de Colombia 1991. Bogotá: Presidencia de la República; 1991.

58. Colombia. El Congreso de Colombia. Ley Estaturaria 1751 de 2015 por medio de la cual se regula el derecho fundamental a la salud y se dictan otras disposiones. Diario Oficial 2015; 16 feb.

59. Ministerio de Salud y Protección Social. Política Nacional de Sexualidad, Derechos Sexuales y Derechos Reproductivos. Bogotá: Misterio de Salud y Protección Social; 2014.

60. Mignone J, Bartlett J, O'Neil J, Orchard T. Best practices in intercultural health: five case studies in Latin America. J Ethnobiol Ethnomed 2007; 3:31.

Recibido el 04/Feb/2015

Versión final presentado el 15/May/2015

Aprobado el 08/Jun/2015 